\title{
Transreal logical space of all propositions
}

Book or Report Section

Accepted Version

dos Reis, T. S. and Anderson, J. (2015) Transreal logical space of all propositions. In: Kim, H. K., Amouzegar, M. A. and Ao, S.-I. (eds.) Transactions on Engineering Technologies, World Congress on Engineering and Computer Science 2014. Springer Netherlands, pp. 227-242. doi:

https://doi.org/10.1007/978-94-017-7236-5 Available at https://centaur.reading.ac.uk/43185/

It is advisable to refer to the publisher's version if you intend to cite from the work. See Guidance on citing.

To link to this article DOI: http://dx.doi.org/10.1007/978-94-017-7236-5

Publisher: Springer Netherlands

All outputs in CentAUR are protected by Intellectual Property Rights law, including copyright law. Copyright and IPR is retained by the creators or other copyright holders. Terms and conditions for use of this material are defined in the End User Agreement.

www.reading.ac.uk/centaur

\section{CentAUR}

Central Archive at the University of Reading 
Reading's research outputs online 


\title{
Transreal Logical Space of All Propositions
}

\author{
Walter Gomide, Tiago S. dos Reis, James A. D. W. Anderson
}

February 23, 2015

\begin{abstract}
Transreal numbers provide a total semantics containing classical truth values, dialetheaic, fuzzy and gap values. A paraconsistent Sheffer Stroke generalises all classical logics to a paraconsistent form. We introduce logical spaces of all possible worlds and all propositions. We operate on a proposition, in all possible worlds, at the same time. We define logical transformations, possibility and necessity relations, in proposition space, and give a criterion to determine whether a proposition is classical. We show that proofs, based on the conditional, infer gaps only from gaps and that negative and positive infinity operate as bottom and top values.

Keywords: all possible worlds, logical spaces, multi-valued logics, paraconsistent logics, transreal numbers, total semantics.

Walter Gomideis with the Philosophy Department; Institute of Humanities and Social Sciences; Federal University of Mato Grosso; 78060-900; Brazil and Program of History of Science, Technique and Epistemology; Federal University of Rio de Janeiro; 21941-916; Brazil

Tiago S. dos Reis is with the: Federal Institute of Education, Science and Technology of Rio de Janeiro; 27215-350; Brazil and Program of History of Science, Technique and Epistemology; Federal University of Rio de Janeiro; 21941-916; Brazil.

James A. D. W. Anderson is with the: School of Systems Engineering; University of Reading; Whiteknights; Reading; England; RG6 6AY.
\end{abstract}

\section{Introduction}

We rehearse our development of total semantics [8] by considering paraconsistent logics. These were explicitly introduced in the second half of the twentieth century as non-classical logics that can reason about inconsistent axioms [12][21]. In a classical logic, inconsistent axioms explode, allowing any theorem to be proved in a trivial way [18][11]. Paraconsistent logics do not explode, they allow only limited conclusions to be drawn from inconsistent axioms. Some admit dialetheias, that is propositions that are both False and True [20], and some admit Gap values with no degree of falsity or truthfulness [23]. Gap values are usually treated absorptively so that any logical combination with a Gap produces a Gap as result. This behaviour is consistent with one reading [17] of Frege's principle of compositionallity so that a compound proposition lacks reference if any component of it lacks reference. It should be added that paraconsistent logics are also capable of classical reasoning so they provide a robust generalisation of classical logic. 
This makes them interesting both from a theoretical and a practical perspective [10][21][28].

Paraconsistent logics are often formalised in advanced mathematics [12][23][24]. We take the simpler approach of expressing them arithmetically. We use transreal arithmetic, which is a generalisation of real arithmetic. Transreal arithmetic was originally developed [1][2] from a subset of the algorithms used in the arithmetic of fractions. It has been axiomatised and a machine proof of consistency has been given [7]. A human proof of consistency is given in [13]. The algorithms of transreal arithmetic are explained, particularly clearly, in a tutorial in [4].

In Section 2 we generalise all classical logics to a paraconsistent form by expressing the Sheffer Stroke [9] in transreal arithmetic. In Section 3 we introduce a logical space in which we operate on a proposition in all possible worlds at the same time. The idea of logical space is inspired by Wittgenstein's conception that the world's logical form is given by a picture that is a "configuration of objects." See [26][27] sections 2, 3 and, especially 3.4. Thus, just as physical objects are arranged in physical space, so logical objects are arranged in a "logical space" [15]. Wittgenstein did not define precisely his notion of logical space. However, by following the intuitive idea that the elements of this space are propositions and the interactions between them are connectives, we establish a logical space as a welldefined mathematical structure, something like a vector space, where the propositions are "vectors" and the connectives are "vector" transformations. In this space we give a mathematical sense to the notion of logical transformation and of possibility and necessity relations. The mathematical treatment ends by establishing a criterion for determining whether a proposition is or is not classical in a given possible world.

\section{Paraconsistent Logic}

We use the entire set of transreal numbers to supply the semantic values, that is the truth values, of paraconsistent logic. In this section we exploit the intuition that a conclusion departs no further from being equally false and true than the most extreme of its antecedents. This is sufficient to make a logic non-explosive. We define a paraconsistent version of the Sheffer Stroke via the minimum value of its antecedents. This paraconsistent operator may be used to generalise all classical logics to a paraconsistent form.

\subsection{Truth Values}

The transreal numbers are just the real numbers augmented with three non-finite numbers: negative infinity $(-\infty)$, positive infinity $(\infty)$ and nullity $(\Phi)$. Nullity is absorptive over the elementary arithmetical operations so that when it is involved in a sum, difference, product or quotient, the result is nullity. However nullity is not universally absorptive, it may be an element of arbitrary mappings. Nullity is the only unordered number in transreal arithmetic [7][6]. Nullity's absorptive properties make it a good candidate for a Gap value that has no degree of falsity or truthfulness [25][8]. We define that negative infinity is classical False and positive

infinity is classical True. This has the merit that we have now used up all of the non-finite, transreal numbers, leaving all of the real numbers to convey dialetheaic 
degrees of falsity and truthfulness. Here we use arithmetical negation (unary subtraction) to model logical negation. Alternative encodings are discussed in [16][8].

Returning now to our paraconsistent logic, we define that the real numbers encode degrees of both falsity and truthfulness. The negative real numbers are more False than True, the positive, real numbers are more True than False, zero is equally False and True. We relate the degree of falsity and truthfulness monotonically to the number modelling the truth value so that negative infinity is entirely False, that is classically False, and positive infinity is entirely True, that is classically True.

\subsection{Sheffer Stroke}

It is known that the truth functional (Boolean) operators for logical negation (not, $\neg$ ), logical conjunction (and, \&), and logical disjunction (or, $\vee$ ) are functionally complete [9] (See entry "Sheffer Stroke" ), [22] (p. 29) so that any truth functional operators can be derived from these three. In fact it is known that the sets $\{\neg, \&\}$ and $\{\neg, \vee\}$ are each functionally complete but it serves our purpose better to consider the wider set of operators $\{\neg, \&, \vee\}$. We use the transreal minimum and maximum functions to define paraconsistent versions of the classical negation, conjunction and disjunction operators. We use negative infinity $(-\infty)$ to model classical False $(\mathrm{F})$ and positive infinity $(\infty)$ to model classical True $(\mathrm{T})$. We use nullity $(\Phi)$ to model the logical Gap value $(\mathrm{G})$. Note that only the real numbers model dialetheaic truth values. The three non-finite numbers each model a single truth value. We then prove that the paraconsistent operators contain the classical ones. With a little extra work we prove that the paraconsistent operators are well defined for all transreal arguments when we assume that the finite, truth values are arranged monotonically with the real numbers that model them. We then define a paraconsistent version of the Sheffer Stroke $(\mid)$. There are three, well known, identities that relate the classical Sheffer Stroke to classical negation, conjunction and disjunction. We show that these identities hold when we substitute the paraconsistent Sheffer Stroke and the paraconsistent negation, conjunction and disjunction. Thus we prove that the paraconsistent operators are defined everywhere and are consistent with their classical counterparts.

We begin by defining the binary, transreal, minimum and maximum functions so that the minimum of two transreal numbers is the least, ordered one of them or else is nullity. Similarly the maximum of two transreal numbers is the greatest, ordered one of them or else is nullity. These definitions rely on the three transreal relations less-than, equal-to, greater-than as axiomatised in [7], explicated in [5] and corrected in [14]. It is sufficient for the reader to know that: nullity is the uniquely unordered, transreal number so it is the only transreal number that compares not-less-than, not-equal-to and not-greater than any other distinct number; negative infinity is the least, ordered, transreal number; positive infinity is the greatest, ordered, transreal number.

Definition 1 Transreal minimum,

$$
\min (a, b)=\left\{\begin{array}{ccc}
a & : & a<b \\
a & : & a=b \\
a & : & b=\Phi \\
b & : & b<a \\
b & : & a=\Phi
\end{array}\right.
$$


Definition 2 Transreal maximum,

$$
\max (a, b)=\left\{\begin{array}{ccc}
a & : & a>b \\
a & : & a=b \\
a & : & b=\Phi \\
b & : & b>a \\
b & : & a=\Phi
\end{array} .\right.
$$

The minimum and maximum functions, just defined, treat nullity non-absorptively but we chose to treat the logical Gap value absorptively.

Definition 3 Paraconsistent conjunction,

$$
a \& b=\left\{\begin{array}{lll}
\Phi & : & a=\Phi \text { or } b=\Phi \\
\min (a, b) & : & \text { otherwise }
\end{array} .\right.
$$

Definition 4 Paraconsistent disjunction,

$$
a \vee b=\left\{\begin{array}{lll}
\Phi & : & a=\Phi \text { or } b=\Phi \\
\max (a, b) & : & \text { otherwise }
\end{array} .\right.
$$

We now define the paraconsistent, logical negation as transarithmetical negation.

Definition 5 Paraconsistent negation, $\neg a=-a$.

Transreal arithmetic has $-0=0,-\Phi=\Phi$ and in all other cases, the negation is distinct so that $-a \neq a$.

The Sheffer Stroke (|) may be defined as an infix operator but we follow the more modern practice of taking it as a post-fix operator so that no bracketing is needed. This leads to shorter and clearer formulas.

Definition 6 Paraconsistent Sheffer Stroke, $a b \mid=\neg(a \& b)$, with all symbols read paraconsistently.

We now prove that the paraconsistent negation, conjunction and disjunction contain their classical counterparts and that the paraconsistent operators are well defined for all transreal arguments.

Theorem 1 Paraconsistent negation contains classical negation.

Proof 1 Classical negation has $\neg \mathrm{F}=\mathrm{T}$ and $\neg \mathrm{T}=\mathrm{F}$. Equivalently paraconsistent negation has $\neg(-\infty)=-(-\infty)=\infty$ and $\neg \infty=-\infty$.

Theorem 2 Paraconsistent conjunction contains classical conjunction.

Proof 2 Classical conjunction has $\mathrm{F} \& \mathrm{~F}=\mathrm{F} ; \mathrm{F} \& \mathrm{~T}=\mathrm{F} ; \mathrm{T} \& \mathrm{~F}=\mathrm{F} ; \mathrm{T} \& \mathrm{~T}=$ T. Equivalently paraconsistent conjunction has $-\infty \&-\infty=\min (-\infty,-\infty)=$ $-\infty ;-\infty \& \infty=\min (-\infty, \infty)=-\infty ; \infty \&-\infty=\min (\infty,-\infty)=-\infty ; \infty \& \infty=$ $\min (\infty, \infty)=\infty$.

Theorem 3 Paraconsistent disjunction contains classical disjunction.

Proof 3 Classical disjunction has $\mathrm{F} \vee \mathrm{F}=\mathrm{F} ; \mathrm{F} \vee \mathrm{T}=\mathrm{T}$; $\mathrm{T} \vee \mathrm{F}=\mathrm{T}$; $\mathrm{T} \vee \mathrm{T}=$ T. Equivalently paraconsistent disjunction has $-\infty \vee-\infty=\max (-\infty,-\infty)=$ $-\infty ;-\infty \vee \infty=\max (-\infty, \infty)=\infty ; \infty \vee-\infty=\max (\infty,-\infty)=\infty ; \infty \vee \infty=$ $\max (\infty, \infty)=\infty$. 
Theorem 4 Paraconsistent negation, conjunction, and disjunction are well defined for all transreal arguments.

Proof 4 Paraconsistent negation, conjunction, and disjunction are defined for all transreal arguments. It remains only to show that these operators are monotonic. Firstly nullity is absorptive in these operators so that if any argument is nullity the result is nullity. Nullity is disjoint from all other transreal numbers because it is the uniquely isolated point of transreal space [6], therefore nullity results are disjoint from all other transreal results and cannot contradict them. Secondly the preceding three theorems show that the paraconsistent operators are well defined at the boundaries $-\infty$ and $\infty$ but, by definition, the non-nullity, paraconsistent, truth values are monotonic so the operators just defined are monotonic for all transreal $t$ in the range $-\infty \leq t \leq \infty$. This completes the proof for all transreal arguments.

We now derive the paraconsistent negation, conjunction and disjunction from formulas involving the paraconsistent Sheffer Stroke. This proves that the paraconsistent Sheffer Stroke is functionally complete both for classical truth values and for the paraconsistent truth values defined here.

Theorem $5 p p \mid=\neg p$ for all transreal $p$.

Proof $5 p p \mid=\neg(p \& p)=\neg p$, with all symbols read paraconsistently.

Theorem $6 p q \mid p q \|=p \& q$ for all transreal $p, q$.

Proof $6 p q|p q||=(\neg(p \& q))(\neg(p \& q))|=\neg(\neg(p \& q))=p$ \& $q$, with all symbols read paraconsistently.

Theorem $7 \quad p p \mid q q \|=p \vee q$ for all transreal $p, q$.

Proof $7 p p|q q||=(\neg p)(\neg q)|=\neg((\neg p) \&(\neg q))=p \vee q$ by the classical de Morgan's Law, generalised to all transreal numbers by monotonicity and the $a b-$ sorptiveness of nullity, with all symbols read paraconsistently.

\section{Proposition Space}

We define logical space, very generally, as a scalar space whose axes are logical elements and whose scalar values are semantic values. This allows us to apply many mathematical methods to logic. We begin with an orthogonal co-ordinate frame where each axis is a copy of the transreal number line. This gives us a trans-Cartesian co-ordinate frame. The more abstract Proposition space has each possible world as an axis and each point is a proposition whose co-ordinates are the semantic values of that proposition in each possible world. This allows us to apply mathematical and logical operations, simultaneously, to propositions in all possible worlds.

We define logical transformations, very generally, as a transformations in logical space. In Section 2, above, we use a paraconsistent Sheffer Stroke. This transformation can be summarised by the side condition that its conclusion departs no further from being equally False and True than its antecedents. We now use a generalisation of the classical conditional which has the property that its conclusion is at least as true as its antecedents. We say that a proposition, or other point, $p$, is derived from a proposition, or other point, $q$, if and only if there is a chain of logical transformations that maps $q$ onto $p$. In particular the chain of conditionals is a proof path. 


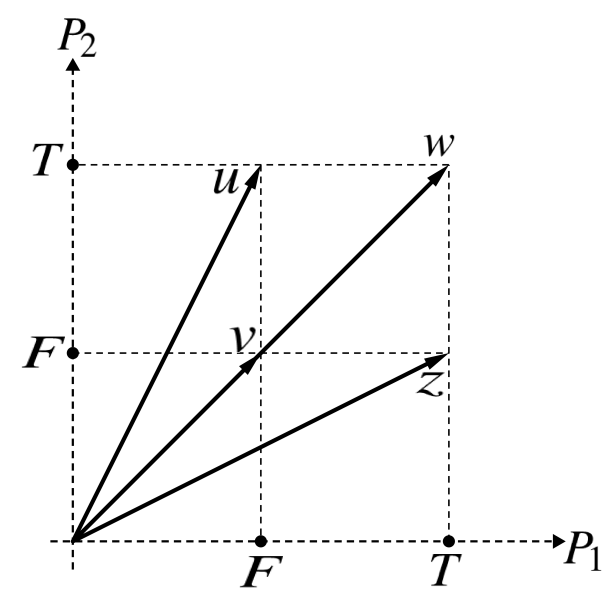

Figure 1: Possible worlds are vectors with a co-ordinate in each proposition. Here $u=(F, T), v=(F, F), w=(T, T), z=(T, F)$ are vectors.

\subsection{Transreal Functions of All Propositions}

We will define atomic propositions as objects in our transreal model. However, to begin, we assume that the set of atomic propositions is a countable set. Hence the set of atomic propositions can be written in the form $\left\{P_{1}, P_{2}, P_{3}, \ldots\right\}$, where $P_{i} \neq P_{j}$ whenever $i \neq j$. Note that we are not defining the set of atomic propositions yet. This is just a representation of the set to be defined.

A possible world is a binding of the atomic propositions to its semantic values. That is, at a given possible world, each atomic proposition takes on a semantic value in $\mathbb{R}^{T}$. Thus we can interpret a possible world as a function from $\left\{P_{1}, P_{2}, P_{3}, \ldots\right\}$ to $\mathbb{R}^{T}$. But this is nothing more than an infinite sequence of elements from $\mathbb{R}^{T}$. In this way, every possible world is an element from $\left(\mathbb{R}^{T}\right)^{\mathbb{N}}$. Conversely every element from $\left(\mathbb{R}^{T}\right)^{\mathbb{N}}$ is a possible world.

To facilitate understanding of possible worlds let us introduce a simplified model with just two semantic values $F$ and $T$ and just two atomic propositions $P_{1}$ and $P_{2}$. An example of a possible world is the world where the atomic proposition $P_{1}$ has semantic value $F$ and the atomic proposition $P_{2}$ has semantic value $T$. Another example is the world where both the atomic propositions $P_{1}, P_{2}$ have semantic value $F$. The first world can be represented by the pair $(F, T)$ and the second world by the pair $(F, F)$, where the first co-ordinate of the pair represents the semantic value of the proposition $P_{1}$ and the second co-ordinate represents the semantic value of the proposition $P_{2}$. In this simplified model there are four possible worlds: $(F, T),(F, F),(T, T)$ and $(T, F)$. These pairs can be viewed geometrically as "vectors:"

Returning to our transreal model, possible worlds are also "vectors" but with infinitely many co-ordinates, not just two, and these co-ordinates take values in $\mathbb{R}^{T}$ not in $\{F, T\}$. Possible worlds are points in $\left(\mathbb{R}^{T}\right)^{\mathbb{N}}$ whose axes are atomic propositions and whose co-ordinates are the semantic value of the underlying atomic proposition in that possible world. Given a possible world $w=\left(w_{i}\right)_{i \in \mathbb{N}} \in\left(\mathbb{R}^{T}\right)^{\mathbb{N}}$, we have that $w_{i}$ corresponds to the semantic value of $P_{i}$ in $w$, for each $i \in \mathbb{N}$. 
We now define atomic propositions in our simplified model, before generalising them in our transreal model. We can represent all possible worlds in a table.

$\begin{array}{ccc}\begin{array}{c}\text { Generic table } \\ \text { (cells) }\end{array} & \begin{array}{c}\text { Possible worlds } \\ \text { (rows) }\end{array} & \begin{array}{c}\text { Atomic propositions } \\ \text { (columns) }\end{array}\end{array}$

\begin{tabular}{||c||c|c||}
\hline \hline & $P_{1}$ & $P_{2}$ \\
\hline \hline$u$ & $F$ & $T$ \\
\hline$v$ & $F$ & $F$ \\
\hline$w$ & $T$ & $T$ \\
\hline$z$ & $T$ & $F$ \\
\hline
\end{tabular}

\begin{tabular}{||c||cc||}
\hline \hline & $P_{1}$ & $P_{2}$ \\
\hline \hline$u$ & $F$ & $T$ \\
\hline$v$ & $F$ & $F$ \\
\hline$w$ & $T$ & $T$ \\
\hline$z$ & $T$ & $F$ \\
\hline \hline
\end{tabular}

\begin{tabular}{||c||c|c||}
\hline \hline & $P_{1}$ & $P_{2}$ \\
\hline \hline$u$ & $F$ & $T$ \\
$v$ & $F$ & $F$ \\
$w$ & $T$ & $T$ \\
$z$ & $T$ & $F$ \\
\hline
\end{tabular}

The generic table associates propositions with worlds. A possible world is a row of the table which gives the semantic values of successive propositions. An atomic proposition is uniquely determined by its semantic values in all possible worlds. That is, if we know the semantic values of an atomic proposition in each possible world, we know this atomic proposition. In other words, an atomic proposition is completely determined by a column of the table. Thus $P_{1}=(F, F, T, T)$ and $P_{2}=(T, F, T, F)$. Here the atomic propositions are 4-tuples, which is to say they are "vectors" of four co-ordinates. Of course we cannot have a picture of the atomic propositions as vectors, because this figure would be in four dimensions, but we can draw the projections in three dimensions, ignoring the fourth co-ordinate. Thus the projections of $P_{1}$ and $P_{2}$, in three dimensions, are $(F, F, T)$ and $(T, F, T)$. Pictorially:

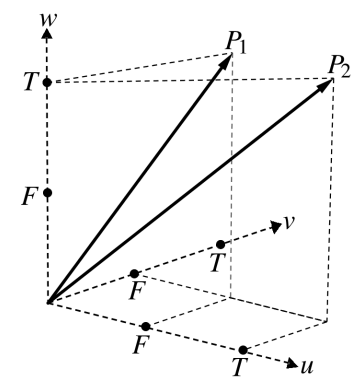

Figure 2: Atomic propositions are vectors with a co-ordinate in each possible world. Here the projections $P_{1}=(F, F, T)$ and $P_{2}=(T, F, T)$ are vectors.

We now extend the simplified model to the transreal model. For each $i \in \mathbb{N}$, let $p_{i}$ be the co-ordinate function $p_{i}:\left(\mathbb{R}^{T}\right)^{\mathbb{N}} \longrightarrow \mathbb{R}^{T}$ where $p_{i}\left(\left(w_{j}\right)_{j \in \mathbb{N}}\right)=w_{i}$. Given $i \in \mathbb{N}$, notice that for each possible world $w=\left(w_{j}\right)_{j \in \mathbb{N}}$, we interpret $p_{i}(w)$ as the semantic value of the $i$-th atomic proposition, $P_{i}$, in the possible world $w$. Hence for each $i \in \mathbb{N},\left(p_{i}(w)\right)_{w \in\left(\mathbb{R}^{T}\right)^{\mathbb{N}}}$ is an $\left(\mathbb{R}^{T}\right)^{\mathbb{N}}$-tuple which expresses the semantic value of the atomic proposition $P_{i}$ in all possible worlds. In this way, each atomic proposition, $P_{i}$, is represented by $\left(p_{i}(w)\right)_{w \in\left(\mathbb{R}^{T}\right)^{\mathbb{N}}}$. This motivates the following definition. 
Definition 7 Let $\mathscr{P}:=\left\{\left(p_{1}(w)\right)_{w \in\left(\mathbb{R}^{T}\right)^{\mathbb{N}}},\left(p_{2}(w)\right)_{w \in\left(\mathbb{R}^{T}\right)^{\mathbb{N}}},\left(p_{3}(w)\right)_{w \in\left(\mathbb{R}^{T}\right)^{\mathbb{N}}}, \ldots\right\}$. We call each element from $\mathscr{P}$ an atomic proposition, hence $\mathscr{P}$ is the set of atomic propositions.

The set $\mathscr{P}$ is infinite. Because the $\left(\mathbb{R}^{T}\right)^{\mathbb{N}}$-tuples

$$
\left(p_{1}(w)\right)_{w \in\left(\mathbb{R}^{T}\right)^{\mathbb{N}}},\left(p_{2}(w)\right)_{w \in\left(\mathbb{R}^{T}\right)^{\mathbb{N}}},\left(p_{3}(w)\right)_{w \in\left(\mathbb{R}^{T}\right)^{\mathbb{N}}}, \cdots
$$

are pairwise distinct. Indeed for each $i, j \in \mathbb{N}$, such that $i \neq j$, there is $u \in\left(\mathbb{R}^{T}\right)^{\mathbb{N}}$ such that $p_{i}(u) \neq p_{j}(u)$. Thus $\left(p_{i}(w)\right)_{w \in\left(\mathbb{R}^{T}\right)^{\mathbb{N}}} \neq\left(p_{j}(w)\right)_{w \in\left(\mathbb{R}^{T}\right)^{\mathbb{N}}}$ whenever $i \neq j$.

By Definition 7, each atomic proposition is a point within the space $\left(\mathbb{R}^{T}\right)^{\left(\mathbb{R}^{T}\right)^{\mathbb{N}}}$, the set of the functions whose domain is the space of sequences of transreal numbers and whose counter-domain is the set of transreal numbers. Further, for each $i \in \mathbb{N},\left(p_{i}(w)\right)_{w \in\left(\mathbb{R}^{T}\right)^{\mathbb{N}}}$ corresponds to $i$-th atomic proposition, that is, $\left(p_{i}(w)\right)_{w \in\left(\mathbb{R}^{T}\right)^{\mathbb{N}}}$ corresponds to $P_{i}$. And, for each $w \in\left(\mathbb{R}^{T}\right)^{\mathbb{N}}, p_{i}(w)$ corresponds to the semantic value of $P_{i}$ in the possible world $w$.

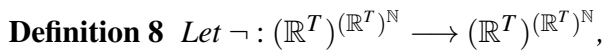

$$
\begin{aligned}
& \neg\left(p_{w}\right)_{w \in\left(\mathbb{R}^{T}\right)^{\mathbb{N}}}=\left(\neg p_{w}\right)_{w \in\left(\mathbb{R}^{T}\right)^{\mathbb{N}}},
\end{aligned}
$$

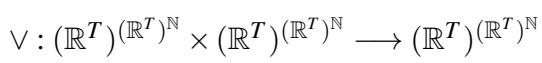

$$
\begin{aligned}
& \left(p_{w}\right)_{w \in\left(\mathbb{R}^{T}\right)^{\mathbb{N}}} \vee\left(q_{w}\right)_{w \in\left(\mathbb{R}^{T}\right)^{\mathbb{N}}}=\left(p_{w} \vee q_{w}\right)_{w \in\left(\mathbb{R}^{T}\right)^{\mathbb{N}}}
\end{aligned}
$$

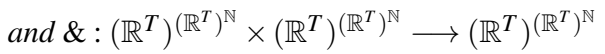

$$
\begin{aligned}
& \left(p_{w}\right)_{w \in\left(\mathbb{R}^{T}\right)^{\mathbb{N}}} \&\left(q_{w}\right)_{w \in\left(\mathbb{R}^{T}\right)^{\mathbb{N}}}=\left(p_{w} \& q_{w}\right)_{w \in\left(\mathbb{R}^{T}\right)^{\mathbb{N}}} .
\end{aligned}
$$

We abuse notation, above, but the reader will perceive that, in (1), the symbol $\neg$, on the left hand side of the equality, refers to a function which is being defined on $\left(\mathbb{R}^{T}\right)^{\left(\mathbb{R}^{T}\right)^{\mathbb{N}}}$, while the symbol $\neg$, on the right hand side of the equality, refers to a function already defined on $\mathbb{R}^{T}$. Similarly for $\vee$ in (2) and for $\&$ in (3).

Definition 9 Let $A \subset\left(\mathbb{R}^{T}\right)^{\left(\mathbb{R}^{T}\right)^{\mathbb{N}}}$ and let $\mathscr{L}_{A}$ be defined as the class of all sets

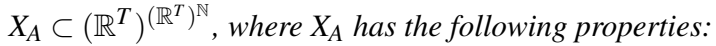

i) $A \subset X_{A}$ and

ii) if $p, q \in X_{A}$ then $\neg p, p \vee q, p \& q \in X_{A}$.

Define $L_{A}:=\bigcap_{X_{A} \in \mathscr{L}_{A}} X_{A}$. Let $p \in\left(\mathbb{R}^{T}\right)^{\left(\mathbb{R}^{T}\right)^{\mathbb{N}}}$ then we say that $p$ is a logical com-

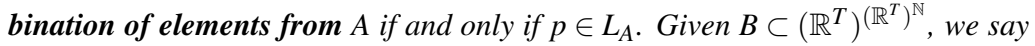
that $B$ is a logically independent set, if and only if, for all $p \in B, p$ is not a logical combination of elements from $B \backslash\{p\}$.

Proposition 1 The set $\mathscr{P}$ is logically independent.

Proof 8 Suppose $\mathscr{P}$ is not logically independent. This means there is an element from $\mathscr{P}$ which is a logical combination of some other elements from $\mathscr{P}$. Without loss of generality, suppose $\left(p_{1}(w)\right)_{w \in\left(\mathbb{R}^{T}\right)^{\mathbb{N}}}$ is a logical combination of some el-

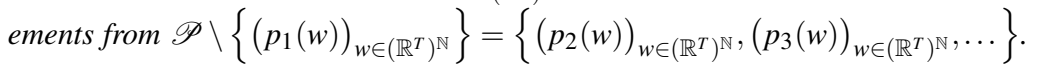


So $\left(p_{1}(w)\right)_{w \in\left(\mathbb{R}^{T}\right)^{\mathbb{N}}}$ is the result of a determinate composition, $T$, between the functions $\neg, \vee$ and \&, applied to some elements from $\left\{\left(p_{2}(w)\right)_{w \in\left(\mathbb{R}^{T}\right)^{\mathbb{N}}},\left(p_{3}(w)\right)_{w \in\left(\mathbb{R}^{T}\right)^{\mathbb{N}}}, \ldots\right\}$. For some $m \in \mathbb{N}, \quad\left(p_{j_{1}}(w)\right)_{w \in\left(\mathbb{R}^{T}\right)^{\mathbb{N}}}, \quad\left(p_{j_{2}}(w)\right)_{w \in\left(\mathbb{R}^{T}\right)^{\mathbb{N}}}, \cdots, \quad\left(p_{j_{m}}(w)\right)_{w \in\left(\mathbb{R}^{T}\right)^{\mathbb{N}}}$ are elements from $\left\{\left(p_{2}(w)\right)_{w \in\left(\mathbb{R}^{T}\right)^{\mathbb{N}}},\left(p_{3}(w)\right)_{w \in\left(\mathbb{R}^{T}\right)^{\mathbb{N}}}, \ldots\right\}$ where $T$ is applied. Now $\left(p_{1}(w)\right)_{w \in\left(\mathbb{R}^{T}\right)^{\mathbb{N}}}=T\left(\left(p_{j_{1}}(w)\right)_{w \in\left(\mathbb{R}^{T}\right)^{\mathbb{N}}},\left(p_{j_{2}}(w)\right)_{w \in\left(\mathbb{R}^{T}\right)^{\mathbb{N}}}, \ldots,\left(p_{j_{m}}(w)\right)_{w \in\left(\mathbb{R}^{T}\right)^{\mathbb{N}}}\right)=$ $\left(T\left(p_{j_{1}}(w), p_{j_{2}}(w), \ldots, p_{j_{m}}(w)\right)\right)_{w \in\left(\mathbb{R}^{T}\right)^{\mathbb{N}}} \cdot$ That is,

$$
p_{1}(w)=T\left(p_{j_{1}}(w), p_{j_{2}}(w), \ldots, p_{j_{m}}(w)\right) \text { for all } w \in\left(\mathbb{R}^{T}\right)^{\mathbb{N}} .
$$

Let arbitrary $\left(u_{1}, u_{2}, \ldots\right) \in\left(\mathbb{R}^{T}\right)^{\mathbb{N}}, u^{\prime} \neq T\left(u_{j_{1}}, u_{j_{2}}, \ldots, u_{j_{m}}\right), v=\left(v_{1}, v_{2}, v_{3} \ldots\right):=$ $\left(u^{\prime}, u_{2}, u_{3}, \ldots\right)$. We have $v \in\left(\mathbb{R}^{T}\right)^{\mathbb{N}}$ and $v_{1} \neq T\left(v_{j_{1}}, v_{j_{2}}, \ldots, v_{j_{m}}\right)$. Hence $p_{1}(v) \neq$ $T\left(p_{j_{1}}(v), p_{j_{2}}(v), \ldots, p_{j_{m}}(v)\right)$. This contradicts (4). Hence $\mathscr{P}$ is logically independent.

Remark 1 Proposition 1 justifies us in calling the propositions in $\mathscr{P}$ atomic.

Definition 10 Let $\Pi:=L_{\mathscr{P}}$, that is,

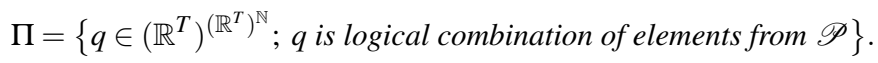

We call $\Pi$ a proposition space and each element from $\Pi$ a proposition.

Proposition 2 The set $\Pi$ is countable.

Proof 9 Each element from $\Pi$ can be written as a finite sequence of symbols from $S:=\mathscr{P} \cup\{\neg, \vee, \&,()$,$\} . Hence an element from \Pi$ can be seen as an element from $S^{n}$ for some $n \in \mathbb{N}$. Thus $\Pi$ can be seen as a subset of $\bigcup_{n \in \mathbb{N}} S^{n}$. Since $S$ is countable, $\bigcup S^{n}$ is countable, whence $\Pi$ is countable. $n \in \mathbb{N}$

Corollary 1 Proposition space, $\Pi$, is a proper subset of $\left(\mathbb{R}^{T}\right)^{\left(\mathbb{R}^{T}\right)^{\mathbb{N}}}$. Thus $\Pi$ is

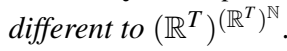

Proof 10 Denote the cardinality of a set $S$ by $|S|$ and let $\mathfrak{c}$ be the cardinality of the continuum. Note that, using Cantor's transfinite arithmetic, $|\Pi|=\aleph_{0}$ but $\left|\left(\mathbb{R}^{T}\right)^{\left(\mathbb{R}^{T}\right)^{\mathbb{N}}}\right|=\mathfrak{c}^{\left(\mathfrak{c}^{\aleph_{0}}\right)}=\mathfrak{c}^{\mathfrak{c}}=\left(2^{\aleph_{0}}\right)^{\mathfrak{c}}=2^{\aleph_{0} \times \mathfrak{c}}=2^{\mathfrak{c}}>\aleph_{0}$. Hence $|\Pi|<\left|\left(\mathbb{R}^{T}\right)^{\left(\mathbb{R}^{T}\right)^{\mathbb{N}}}\right|$

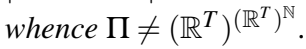

Remark 2 Notice that every proposition, in the ordinary sense, lies in $\Pi$ but $\Pi$ is within the bigger set $\left(\mathbb{R}^{T}\right)^{\left(\mathbb{R}^{T}\right)^{\mathbb{N}}}$. Hence there are points from $\left(\mathbb{R}^{T}\right)^{\left(\mathbb{R}^{T}\right)^{\mathbb{N}}}$ that are not logical combinations of atomic propositions. These points are not expressible in any language but this does not require that they are meaningless. This issue

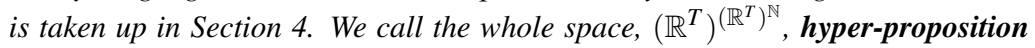
space.

Remark 3 We emphasise that in our transreal model there are three distinct sets of propositions:

- $\mathscr{P}$ is the set of all atomic propositions. 
- П, called proposition space, is the set of all propositions (atomic or molecular).

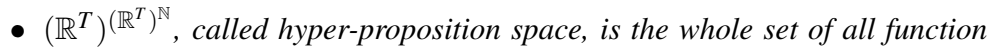
whose domain is the space of sequences of transreal numbers and whose counter-domain is the set of transreal numbers.

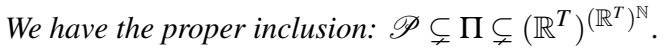

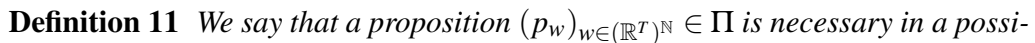
ble world $u$ if and only if $p_{w}>0$ for all $w \in\left(\mathbb{R}^{T}\right)^{\mathbb{N}}$, such that $u$ accesses $w$. And

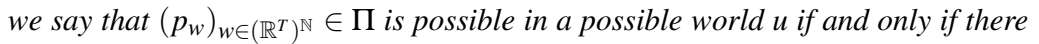
is a $w \in\left(\mathbb{R}^{T}\right)^{\mathbb{N}}$ such that $u$ accesses $w$ and $p_{w}>0$. A world $u$ accesses a world $w$ if and only if there is a linear transformation on $\left(\mathbb{R}^{T}\right)^{\mathbb{N}}$ that maps u onto $w$.

\subsection{Logical Transformations}

In classical logic the connective conditional, $\rightarrow$, is defined as follows [19]:

$$
\begin{aligned}
& \rightarrow:\{F, T\} \times\{F, T\} \longrightarrow\{F, T\} \\
& F \rightarrow F=T, \quad F \rightarrow T=T \\
& T \rightarrow F=F, \quad T \rightarrow T=T
\end{aligned} .
$$

This means that:

i) if the antecedent is false then the conditional is true, regardless of the value of the consequent and

ii) if the consequent is true then the conditional is true, regardless of the value of the antecedent.

In non-classical logics, the conditional is defined in various ways. However, the above observation gives us the familiar intuition that the conditional is true if and only if the degree of truth of the consequent is greater than or equal to the degree of truth of the antecedent. Motivated by this, we propose the following definition.

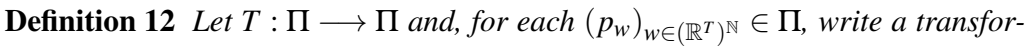

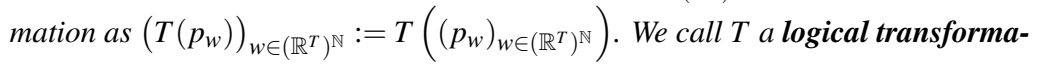
tion if and only if $p_{w} \leq T\left(p_{w}\right)$ for all $w \in\left(\mathbb{R}^{T}\right)^{\mathbb{N}}$.

It is well known that, in classical, propositional calculus, one can derive any proposition from bottom [18][11], $\perp$, that is:

$$
\text { for all } p \text { within the system, } \perp \vdash p \text {. }
$$

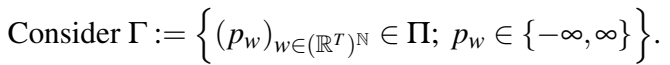

In $\Gamma$, the meta-theorem in (5) is equivalent to an affirmative answer to the question: is there any point in $\Gamma$ from which we can derive, by means of a logical

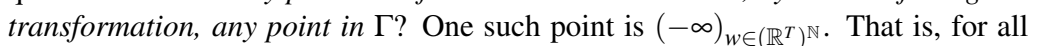

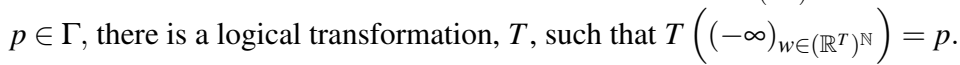

In an analogous way we can read the meta-theorem: one can derive top, $T$,

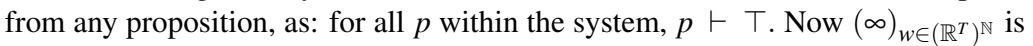
a point which can be derived from any other point, that is: or all $p \in \Gamma$, there is a

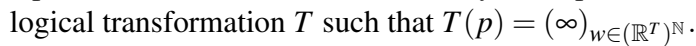


If we consider a propositional calculus in which one allows continuous degrees of truth and falseness, and, furthermore, propositions that can be both true and false then the "bottom-point" still is the point from where every point can be reached and the "top-point" still is the point to which every point can derive. The verification of this is analogous to the classical case but now we consider

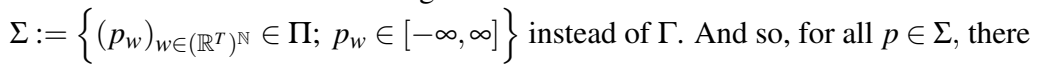

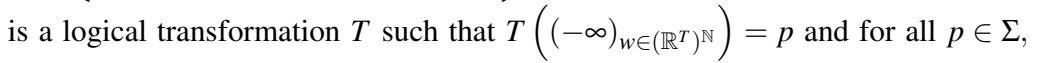
there is a logical transformation $T$ such that $T(p)=(\infty)_{w \in\left(\mathbb{R}^{T}\right)^{\mathbb{N}}}$.

If we extend propositional calculus to $\Pi$ then the "bottom-point" is no longer the "privileged place" from which we can derive any point of $\Pi$. Let $q=\left(q_{w}\right)_{w \in\left(\mathbb{R}^{T}\right)^{\mathbb{N}} \in}$ $\Pi$ such that, for some $u \in\left(\mathbb{R}^{T}\right)^{\mathbb{N}}, q_{u}=\Phi$. The $u$-th co-ordinate of the "bottompoint" is $-\infty$ and, since $-\infty \leq \Phi$ does not hold then we can not derive $q$ from

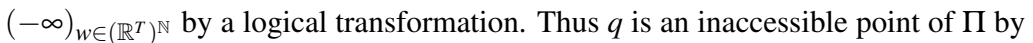
means of a logical transformation that has as initial points those whose co-ordinates belong to $[-\infty, \infty]$. Points like $q$, that have $\Phi$ as the value of some co-ordinate, are only derivable from points whose corresponding co-ordinate is also $\Phi$.

\subsection{A criterion to distinguish classical and non-classical propositions}

Since a proposition is a point in proposition space and since an axis, $u$, of this space is a possible world, if a proposition behaves classically, its numerical value at $u$ is $-\infty$ (classical false) or $\infty$ (classical true). Hence its contradictory is a point in the proposition space that has $u$-co-ordinate $\neg(-\infty)=\infty$ or $\neg(\infty)=-\infty$. Thus if, in a given possible world $u$, a proposition is classical then the absolute difference between the $u$-co-ordinates of the proposition and of its contradictory is $|(-\infty)-(\infty)|$ or $|\infty-(-\infty)|$, whichever case holds $|(-\infty)-(\infty)|=|\infty-(-\infty)|=$ $\infty$. Conversely if the absolute difference between the $u$-co-ordinates of a propo-

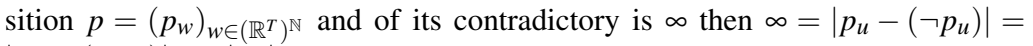
$\left|p_{u}-\left(-p_{u}\right)\right|=2\left|p_{u}\right|$ whence $p_{u}=-\infty$ or $p_{u}=\infty$. Hence $p$ is classical in the possible world $u$. This demonstrates the following proposition.

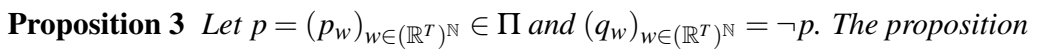
$p$ is classical in the possible world $u \in\left(\mathbb{R}^{T}\right)^{\mathbb{N}}$ if and only if $\left|p_{u}-q_{u}\right|=\infty$.

Thus in our proposition space, which instantiates a total semantics, we stipulate a criterion to distinguish classical from non-classical propositions. Usually there is no way to distinguish atomic propositions because they have no inner structure and, therefore, no feature that can be used as a criterion for the distinction, which is taken arbitrarily. But, as propositions are points located at co-ordinates in proposition space, atomic propositions are elements of a structured space and this structure offers a criterion for distinguishing classical and non-classical atomic propositions.

\section{Discussion}

Proposition space, $\Pi$, is a geometrical version of the usual propositional calculus. It has all the expected, logical properties of paraconsistent, propositional calculi 
and offers a classical structure when it operates on positive and negative infinity, which represent the classical truth values, False and True respectively.

Proposition space, $\Pi$, is a small part of the entire hyper-proposition space,

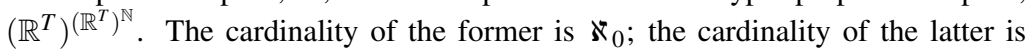
greater than the cardinality of the continuum. Thus we can infer that there are proposition points that will never be expressed by logical combinations of atomic propositions: these points are essentially non-logical, since we understand by "logical" that a point belongs to proposition space. But does this imply that complementary elements, in hyper-proposition space but outside proposition space, are meaningless? We think not.

Complementary points represent what is logically inexpressible: they contain information, since they lie in the entire $\left(\mathbb{R}^{T}\right)^{\left(\mathbb{R}^{T}\right)^{\mathbb{N}}}$ space, but this information cannot be accessed through ordinary, logical reasoning, expressed in any language. Perhaps we should associate, at least some of them, with metaphysical or philosophical statements that cannot be proved by logical apparatus?

On this point let us emphasise the role of logical transformations. In Section 3.2 a logical transformation is defined as a certain transformation in proposition space. If we extend this definition, by allowing transformations in the entire hyperproposition space, then the concept of a continuous proof path appears. Recall that a proof path is a chain of compositions of logical transformations. If we restrict ourselves to the enumerable proposition space then a proof path has a finite or denumerably infinite length but, more generally speaking, a proof path is a geometrical translation of the notion of demonstration that is used in logic: a list of propositions that start with premises, supposed to be true, and a conclusion that is true in virtue of the soundness or correctness of the rules of inference. In the entire hyper-proposition space, this proof path can be continuous. This fact is very significant: it gives us a geometrical entity, a continuous path, that has logical meaning. This path must have a non-denumerable infinitude of hidden propositions - let us call them subatomic propositions. Hence we see the need to expand the notion of a discrete demonstration to a continuous demonstration in which, between two proof steps indexed with finite numbers, there is a continuum of steps that cannot be expressed in language.

A finite simulation (not an emulation) of a machine that operates on a continuum of propositions is given in [3].

\section{Conclusion}

We develop a model for a total semantics, for possible worlds, for proposition space and for hyper-proposition space. We define the set of semantic values as the set of transreal numbers, $\mathbb{R}^{T}$. This is sufficient to model classical truth values, paraconsistent, fuzzy and gap values. We give a paraconsistent version of the Sheffer Stroke which is sufficient to extend all classical logics to a paraconsistent form. We then turn our attention to logical spaces. We define each possible world, in world space, as a sequence of transreal numbers. We define the set of propo-

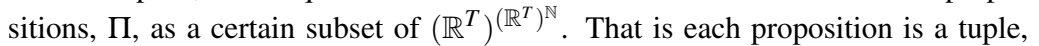

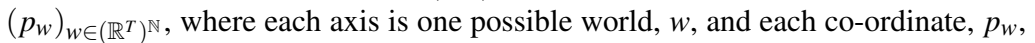
of $p$ is the transreal semantic value of $p$ in the possible world $w$. This allows us to operate on a proposition in all possible worlds at the same time. We define in $\Pi$ the concepts of possibility, necessity and logical transformation and we define a crite- 
rion which distinguishes whether a proposition is or is not classical. A proposition is classical in a determined, possible world if and only if the absolute difference between the semantic value of the proposition and its negation, in the underlying possible world, is infinite. We show that proofs based on the conditional can infer gap values only from gap values, that the proposition which is classically False $(-\infty)$, in all possible worlds, is a bottom point from which all non-gap propositions can be derived and that the proposition which is classically True $(\infty)$, in all possible worlds, is a top point entailed by all non-gap propositions. We discuss the need for continuous versions of logic that capture inferences and proofs in our very high cardinality hyper-proposition space, $\left(\mathbb{R}^{T}\right)^{\left(\mathbb{R}^{T}\right)^{\mathbb{N}}}$.

\section{6 acknowledgement}

The authors would like to thank the members of Transmathematica for many helpful discussions. The third author's research was financially supported, in part, by the School of Systems Engineering at the University of Reading and by a Research Travel Grant from the University of Reading Research Endowment Trust Fund (RETF).

\section{References}

[1] J. A. D. W. Anderson. Representing geometrical knowledge. Phil. Trans. Roy. Soc. Lond. Series B., 352(1358):1129-1139, 1997.

[2] James A. D. W. Anderson. Exact numerical computation of the rational general linear transformations. In Longin Jan Lateki, David M. Mount, and Angela Y. Wu, editors, Vision Geometry XI, volume 4794 of Proceedings of SPIE, pages 22-28, 2002.

[3] James A. D. W. Anderson. Perspex machine iii: Continuity over the turing operations. In Longin Jan Lateki, David M. Mount, and Angela Y. Wu, editors, Vision Geometry XIII, volume 5675 of Proceedings of SPIE, pages 112-123, 2005.

[4] James A. D. W. Anderson. Evolutionary and revolutionary effects of transcomputation. In 2nd IMA Conference on Mathematics in Defence. Institute of Mathematics and its Applications, Oct. 2011.

[5] James A. D. W. Anderson. Trans-floating-point arithmetic removes nine quadrillion redundancies from 64-bit ieee 754 floating-point arithmetic. In Lecture Notes in Engineering and Computer Science: Proceedings of The World Congress on Engineering and Computer Science 2014, WCECS 2014, 22-24 October, 2014, San Francisco, USA., volume 1, pages 80-85, 2014.

[6] James A. D. W. Anderson and Tiago S. dos Reis. Transreal limits expose category errors in ieee 754 floating-point arithmetic and in mathematics. In Lecture Notes in Engineering and Computer Science: Proceedings of The World Congress on Engineering and Computer Science 2014, WCECS 2014, 22-24 October, 2014, San Francisco, USA., volume 1, pages 86-91, 2014.

[7] James A. D. W. Anderson, Norbert Völker, and Andrew A. Adams. Perspex machine viii: Axioms of transreal arithmetic. In Longin Jan Lateki, David M. 
Mount, and Angela Y. Wu, editors, Vision Geometry XV, volume 6499 of Proceedings of SPIE, pages 2.1-2.12, 2007.

[8] James A.D.W. Anderson and Walter Gomide. Transreal arithmetic as a consistent basis for paraconsistent logics. In Lecture Notes in Engineering and Computer Science: Proceedings of The World Congress on Engineering and Computer Science 2014, WCECS 2014, 22-24 October, 2014, San Francisco, USA., volume 1, pages 103-108, 2014.

[9] Robert Audi. The Cambridge Dictionary of Philosophy. Cambridge University Press, 2nd edition, 1997.

[10] R. E. Bellman and L. A. Zadeh. Local and fuzzy logics. In J. M. Dunn and G. Epstein, editors, Modern Uses of Multiple-Valued Logic, pages 103-165. D. Reidel Publishing Co., 1977.

[11] W. Carnelli and J. Marcos. Ex contradictione non sequitur quodlibet. In Proc. 2nd Conf. on Reasoning and Logic, Bucharest, 2001.

[12] N. C. A. da Costa. The philosophical import of paraconsistent logic. Journal of Non-Classical Logic, 1:1-19, 1982.

[13] Tiago S. dos Reis and James A. D. W. Anderson. Construction of the transcomplex numbers from the complex numbers. In Lecture Notes in Engineering and Computer Science: Proceedings of The World Congress on Engineering and Computer Science 2014, WCECS 2014, 22-24 October, 2014, San Francisco, USA., volume 1, pages 97-102, 2014.

[14] Tiago S. dos Reis and James A. D. W. Anderson. Transreal calculus. IAENG International Journal of Applied Mathematics, 45(1):51-63, 2015.

[15] Juliet Floyd. Wittgenstein on philosophy of logic and mathematics. In Stewart Shapiro, editor, Oxford Handbook of Philosophy of Logic and Mathematics, chapter 4, pages 75-128. Oxford University Press, 2005.

[16] Walter Gomide. O princípio de não-contradição e sua tradução para a aritmética transreal. Investigação Filosófica, 4(1), 2013.

[17] Theo M.V. Jansen. Frege, contextuality and composionality. Journal of Logic, language, and Information, 10, 2001.

[18] P. Martin-Löf. On the meanings of the logical constants and the justification of the logical laes. Nordic Journal of Philosophical Logic, 1:11-60, 1996.

[19] John Nolt and Dennis Rohatyn. Logic. McGraw-Hill, 1988.

[20] G. Priest, J. C. Beall, and B. A. Gab. The Law of Non-Contradiction. Oxford University Press, 2004.

[21] Graham Priest, Koji Tanaka, and Zach Weber. Paraconsistent logic. The Stanford Encyclopedia of Philosophy, http://plato.stanford.edu/archives/fall2013/entries/logic-paraconsistent, 2013.

[22] Kenneth H. Rosen. Discrete Mathematics and its Appications. McGraw-Hill, 6th edition, 2007.

[23] Y. Shramko and H. Wansing. Entailment relations and truth values. Bulletin of the Section of Logic, 36:131-143, 2007.

[24] Y. Shramko and H. Wansing. Truth and falsehood. an inquiry into generalised logical values. Trends in Logic, 36, 2007. 
[25] B.C. van Fraasen. Singular terms, truth values, gaps, and frege logic. Journal of Philosophy, 63:481-495, 1966.

[26] Ludwig Wittgenstein. Logisch-philosophische abhandlung. Unesma, 1921.

[27] Ludwig Wittgenstein. Tractatus Logico-Philasophicus. Gutenberg, 2010.

[28] L. A. Zadeh. Fuzzy logic and approximate reasoning. Synthese, 30:407-428, 1975. 